\title{
SOME RESULTS RELATED TO DISTRIBUTION FUNCTIONS OF CHI-SQUARE TYPE RANDOM VARIABLES WITH RANDOM DEGREES OF FREEDOM
}

\author{
Tran loc Hung, Tran Thien Thanh, and But Quang Vu
}

\begin{abstract}
The main aim of this paper is to present some results related to asymptotic behavior of distribution functions of random variables of chi-square type $\chi_{N}^{2}=\sum_{i=1}^{N} X_{i}^{2}$ with degrees of freedom $N$, where $N$ is a positive integer-valued random variable independent on all standard normally distributed random variables $X_{i}$. Two ways for computing the distribution functions of chi-square type random variables with random degrees of freedom are considered. Moreover, some tables concerning considered distribution functions are demonstrated in Appendix.
\end{abstract}

\section{Introduction}

Let $X_{1}, X_{2}, \ldots, X_{n}$ be $n$ independent identically distributed (i.i.d.) random variables of standard normal law $\mathcal{N}(0,1)$. The sum $X_{1}^{2}+X_{2}^{2}+\cdots+X_{n}^{2}$ is said to be a chi-square random variable of $n$ degrees of freedom, denoted by $\chi_{n}^{2}$.

The density function of random variable $\chi_{n}^{2}$ is defined by (we refer the reader to [2] and [3].)

$$
f_{n}(x)=\left\{\begin{array}{lll}
0 & \text { if } & x \leq 0 \\
\frac{x^{\frac{n}{2}}-1}{2^{\frac{n}{2}} \Gamma\left(\frac{n}{2}\right)} e^{-\frac{x}{2}} & \text { if } & x>0
\end{array}\right.
$$

where $\Gamma(s)=\int_{0}^{\infty} e^{-x} x^{s-1} d x$ denotes the Gamma function.

It has long been known that in probability theory and statistics, the chisquare distribution of random variable $\chi_{n}^{2}$ (also chi-squared or $\chi^{2}$-distribution) is one of the most widely used theoretical probability distributions in inferential statistics, for instance in chi-square tests and in estimating variances. This distribution enters the problem of estimating the mean of a normally distributed population and the problem of estimating the slope of a regression line via its

Received October 22, 2007.

2000 Mathematics Subject Classification. 60F05, 60E10, 60G50, 62E20.

Key words and phrases. random sum, chi-square random variable with degrees of freedom.

This work is partially supported by the National Basic Research Program 2006-2008 (MOST, Vietnam) code 101806 and Asia Research Center (VNU, Vietnam) Grant 20072008. 
role in Student's $t$-distribution. Moreover, it also enters all analysis of variance problems via its role in the $F$-distribution, which is the distribution of the ratio of two independent chi-square random variables divided by their respective degrees of freedom (see [2] and [3] for more details).

During the last several decades the random-index approach has risen to become one of the most important tools available for investigating some problems of Applied Statistics (for a deeper discussion of this we refer the reader to [4] and [8]).

The question arises as to what happens if the degrees of freedom $n$ of a chi-square random variable $\chi_{n}^{2}$ shall be replayed by a positive integer-valued random variable $N$. The answer of above question is main aim of this paper. The applications of probability distributions of the random variable of chisquare type $\chi_{N}^{2}$ with random degrees of freedom $N$ in some applied problems of statistics will be taken up in the next research results.

This paper is divided into five main sections. The second section deals with the asymptotic behaviors of distributions of chi-square random variable $\chi_{n}^{2}$ (Theorem 2.1) and of chi-square type $\chi_{N}^{2}$ with random degrees of freedom $N$ (Theorems 2.2-2.8). The third section gives the proofs of all results in second section. The fourth section describes two approaches to computation of the distribution functions of the random variable of chi-square type $\chi_{N}^{2}$ with random degrees of freedom $N$. The Appendix (last section) devotes to some tables concerning the distribution functions of chi-square type $\chi_{N}^{2}$ with random degrees of freedom $N$ in some concrete cases.

\section{Main results}

Throughout this paper, we denote by $Z$ a random variable degenerated at point 1 , and by $\varphi_{Z}(t)=e^{i t}$ its characteristic function.

From now on, the notation $\stackrel{d}{\rightarrow}$ will mean the convergence in distribution and $\stackrel{\mathbb{P}}{\rightarrow}$ will denote the convergence in probability.

Theorem 2.1. Let $\chi_{n}^{2}$ be a chi-square random variable of $n$ degrees of freedom ( $n$ is positive integer number). Then

$$
\frac{\chi_{n}^{2}}{n} \stackrel{d}{\rightarrow} Z
$$

as $n \rightarrow \infty$.

Theorem 2.2. Let $X_{1}, X_{2}, \ldots$ be a sequence of i.i.d. random variables with chi-square distribution function $\chi_{n}^{2}$. Suppose that $N$ is a positive integer-valued random variable independent of all $X_{i}$. Furthermore, let us consider the random sum $S_{N}:=\sum_{i=1}^{N} X_{i}$. Then

as $n \rightarrow \infty$.

$$
\frac{S_{N}}{n} \stackrel{d}{\rightarrow} N
$$


We now return to some interesting results concerning to random variable $\chi_{N_{n}}^{2}:=X_{1}^{2}+\cdots+X_{N_{n}}^{2}$, if for the random variable $\chi_{n}^{2}$ with degrees of freedom $n$, the fixed number $n$ will be replaced by the positive integer-valued random variables $N_{n}, n \geq 1$, independent of all $X_{i} \sim \mathcal{N}(0,1), i=1,2, \ldots, N_{n}$. The following theorems will be main results of this paper.

Theorem 2.3. Let $N_{n} \sim \operatorname{Binomial}(n, p), p \in(0,1)$. Then

as $n \rightarrow \infty$.

$$
\frac{\chi_{N_{n}}^{2}}{n p} \stackrel{d}{\rightarrow} Z
$$

Theorem 2.4. Let $N_{n} \sim \operatorname{Poisson}\left(\lambda_{n}\right), \lambda_{n}>0, n=1,2, \ldots$ and $\lambda_{n} \rightarrow \infty$ as $n \rightarrow \infty$. Then

as $n \rightarrow \infty$.

$$
\frac{\chi_{N_{n}}^{2}}{\lambda_{n}} \stackrel{d}{\rightarrow} Z,
$$

Theorem 2.5. Let $\left\{N_{n}, n \geq 1\right\}$ be a sequence of positive integer-valued random variables, independent from all $X_{i} \sim \mathcal{N}(0,1), i=1,2, \ldots$ Furthermore, assume that the following conditions are satisfied

$$
E\left(N_{n}\right) \rightarrow \infty ; \quad \frac{E\left|N_{n}-E\left(N_{n}\right)\right|}{E\left(N_{n}\right)} \rightarrow 0 \quad \text { as } n \rightarrow \infty .
$$

Then, as $n \rightarrow \infty$ we get

$$
\frac{\chi_{N_{n}}^{2}}{E\left(N_{n}\right)} \stackrel{d}{\rightarrow} Z .
$$

Remark 1. (a) Theorem 2.5 is generalization of Theorems 2.1,2.3, and 2.4.

(b) It is easily seen that $E\left(\chi^{2}(1)\right)=1$. Thus, as a fundamental result from random sum, it follows $E\left(\chi_{N_{n}}^{2}\right)=E\left(\chi^{2}(1)\right) E\left(N_{n}\right)$. Then, (3) in Theorem 2.5 can be formulated as follows

$$
\frac{\chi_{N_{n}}^{2}}{E\left(\chi_{N_{n}}^{2}\right)} \stackrel{d}{\rightarrow} Z, \quad \text { as } n \rightarrow \infty .
$$

Theorem 2.6. Let $N_{n} \sim \operatorname{Uniform}(n)$. Then, as $n \rightarrow \infty$, we have

$$
\frac{\chi_{N_{n}}^{2}}{n} \stackrel{d}{\rightarrow} U \sim \text { Uniform }[0,1] \text {. }
$$

Theorem 2.7. Let $N_{n} \sim \operatorname{Geometric}\left(p_{n}\right)$ and suppose that $p_{n} \rightarrow 0$ as $n \rightarrow \infty$. Then

$$
p_{n} \chi_{N_{n}}^{2} \stackrel{d}{\rightarrow} Y \sim \operatorname{Exp}(1)
$$

as $n \rightarrow \infty$.

Remark 2. According to Theorems 2.6 and 2.7 we can see the condition $E\left(N_{n}\right) \rightarrow \infty$ in (2) (as $n \rightarrow \infty$ ) is not sufficient to confirm conclusion as in Remark 1.b. 
Theorem 2.8. Let $\left\{N_{n}, n \geq 1\right\}$ be a sequence of positive integer-valued random variables, independent from all $X_{i} \sim \mathcal{N}(0,1), i=1,2, \ldots$ Moreover, if

$$
\frac{N_{n}}{n} \stackrel{\mathbb{P}}{\rightarrow} 1, \quad n \rightarrow \infty
$$

then, as $n \rightarrow \infty$, we get

$$
\frac{\chi_{N_{n}}^{2}}{n} \stackrel{d}{\rightarrow} Z
$$

Remark 3. (a) The condition (5) in Theorem 2.8 is Feller's condition (see [1]).

(b) According to the results of paper [7], we will be right in a conjecture: if random variables $N_{n}$ have asymptotic normal distribution function, then the $\chi_{N_{n}}^{2}$ has asymptotic generate distribution function at point one.

\section{Proofs}

Proof of Theorem 2.1. It is easily seen that, the characteristic function of the $\chi_{n}^{2}$ is defined by

$$
\varphi(t)=\frac{1}{(1-2 i t)^{\frac{n}{2}}}=\left(\frac{1+2 i t}{1+4 t^{2}}\right)^{\frac{n}{2}}=\left[1+\frac{2 i t-4 t^{2}}{1+4 t^{2}}\right]^{\frac{n}{2}} .
$$

Then

$$
\varphi_{\frac{\chi_{n}^{2}}{n}}(t)=\varphi(t / n)=\left[1+\frac{2}{n}\left(\frac{i n t-2 t^{2}}{n+4 t^{2} / n}\right)\right]^{\frac{n}{2}} .
$$

By letting $n \rightarrow \infty$, we have $\varphi_{\frac{\chi_{n}^{2}}{n}}(t) \rightarrow e^{i t}$. It follows the proof of theorem.

Proof of Theorem 2.2. Denote by $g$ the generating function of $N$, and by $\varphi$ the characteristic function of $\chi_{n}^{2}$. According to proof of Theorem 2.1 we obtained $\varphi(t / n) \rightarrow e^{i t}$, as $n \rightarrow \infty$. Then, characteristic function of $\frac{S_{N}}{n}$ will be defined by

$$
\varphi_{\frac{S_{N}}{n}}(t)=g(\varphi(t / n)) \rightarrow g\left(e^{i t}\right)=E\left(e^{i t N}\right), \quad \text { as } \quad n \rightarrow \infty .
$$

It completes the proof of theorem.

Proof of Theorem 2.3. Under assumption of theorem, it is easily seen that the random variable $N_{n}$ has generating function $g(t)=[1+p(t-1)]^{n}$ and the random variable $X_{k}^{2}$ has characteristic function $\varphi(t)=\frac{1}{\sqrt{1-2 i t}}$. Then, the characteristic function of $\frac{\chi_{N_{n}}^{2}}{n p}$ is given by

$$
\begin{aligned}
\varphi_{\frac{x_{N n}^{2}}{n p}}^{2} & =g(\varphi(t / n p))=[1+p(\varphi(t / n p)-1)]^{n} \\
& =\left[1+p\left(\frac{\sqrt{n p}}{\sqrt{n p-2 i t}}-1\right)\right]^{n}=\left[1+\frac{1}{n} \frac{2 i t n p}{(\sqrt{n p}+\sqrt{n p-2 i t}) \sqrt{n p-2 i t}}\right]^{n} \\
& =\left[1+\frac{1}{n} \frac{2 i t}{(1+\sqrt{1-2 i t / n p}) \sqrt{1-2 i t / n p}}\right]^{n} .
\end{aligned}
$$




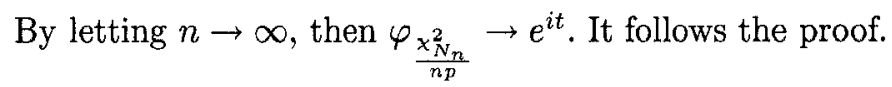

Proof of Theorem 2.4. Obviously, from the assumption of theorem, we can check that the random variable $N_{n}$ has generating function $g(t)=e^{\lambda_{n}(t-1)}$ and the random variable $X_{k}^{2}$ has characteristic function $\varphi(t)=\frac{1}{\sqrt{1-2 i t}}$. Then, characteristic function of $\chi_{N_{n}}^{2} / \lambda_{n}$ is given by

$$
\begin{aligned}
\varphi_{\frac{\chi_{N_{n}}^{2}}{\lambda_{n}}} & =g\left(\varphi\left(t / \lambda_{n}\right)\right)=e^{\lambda_{n}\left[\varphi\left(t / \lambda_{n}\right)-1\right]} \\
& =e^{\lambda_{n}\left[\frac{\sqrt{\lambda_{n}}-\sqrt{\lambda_{n}-2 i t}}{\sqrt{\lambda_{n}}-2 i t}\right]}=e^{\frac{2 i t}{\left(1+\sqrt{1-2 i t / \lambda_{n}}\right) \sqrt{1-2 i t / \lambda_{n}}}} .
\end{aligned}
$$

By letting $n \rightarrow \infty$, we obtain $\varphi_{\frac{\chi_{N_{n}}^{2}}{\lambda_{n}}} \rightarrow e^{i t}$. This completes the proof.

Proof of Theorem 2.5. Put $a_{n}=E\left(N_{n}\right)$ and $p_{k}=P\left(N_{n}=k\right)$. It is easily seen that the generating function of random variable $N_{n}$ is $g(t)=E\left(t^{N_{n}}\right)$. Because of all random variables $X_{k}, k=1,2, \ldots, n$ belong to standard normal law, the characteristic function of random variables $X_{k}^{2}, k=1,2, \ldots n$, is given by $\varphi(t)=\frac{1}{\sqrt{1-2 i t}}$. Then, the characteristic function $\psi_{n}$ of $\frac{\chi_{N_{n}}^{2}}{a_{n}}$ will be given by

$$
\begin{aligned}
\psi_{n}(t) & =g\left(\varphi\left(t / a_{n}\right)\right)=\sum_{k=0}^{\infty} p_{k} \varphi^{k}\left(t / a_{n}\right)=\sum_{k=0}^{\infty} p_{k}\left(1-2 i t / a_{n}\right)^{-k / 2} \\
& =\sum_{k=0}^{\infty} p_{k}\left[1+\frac{2}{a_{n}}\left(\frac{i t-2 t^{2} / a_{n}}{1+4 t^{2} / a_{n}^{2}}\right)\right]^{k / 2} .
\end{aligned}
$$

Putting

We have

$$
\delta_{n}=\left[1+\frac{2}{a_{n}}\left(\frac{i t-2 t^{2} / a_{n}}{1+4 t^{2} / a_{n}^{2}}\right)\right]^{a_{n} / 2}
$$

$$
\left|\delta_{n}\right| \leq 1 ; \quad \delta_{n} \rightarrow e^{i t} \quad \text { as } \quad n \rightarrow \infty
$$

It is easily seen that

$$
\left|\psi_{n}(t)-\delta_{n}\right|=\left|\sum_{k=0}^{\infty} p_{k}\left[\delta_{n}^{k / a_{n}}-\delta_{n}\right]\right| \leq \sum_{k=0}^{\infty} p_{k}\left|\delta_{n}^{k / a_{n}}-\delta_{n}\right| .
$$

Let us consider the continuous function $h(x)=\delta_{n}^{x}$ on $\left[k / a_{n}, 1\right]$ or $\left[1, k / a_{n}\right]$. According to Lagrange's Theorem and (6), we can obtain

$$
\begin{aligned}
\left|\delta_{n}^{k / a_{n}}-\delta_{n}\right| & =\left|h\left(k / a_{n}\right)-h(\mathbf{1})\right|=\left|k / a_{n}-1\right|\left|h^{\prime}(c)\right| \quad(c>0) \\
& =\left|k / a_{n}-1\right|\left|\ln \delta_{n}\right|\left|\delta_{n}\right|^{c} \leq\left|k / a_{n}-1\right|\left|\ln \delta_{n}\right| .
\end{aligned}
$$

Thus, our task is to estimate

$$
\left|\psi_{n}(t)-\delta_{n}\right| \leq \sum_{k=0}^{\infty} p_{k}\left|\ln \delta_{n}\right| \frac{\left|k-a_{n}\right|}{a_{n}}=\left|\ln \delta_{n}\right| \frac{E\left|N_{n}-a_{n}\right|}{a_{n}}
$$


From this we deduce that

$$
\left|\psi_{n}(t)-e^{i t}\right| \leq\left|\psi_{n}(t)-\delta_{n}\right|+\left|\delta_{n}-e^{i t}\right| \leq\left|\ln \delta_{n}\right| \frac{E\left|N_{n}-a_{n}\right|}{a_{n}}+\left|\delta_{n}-e^{i t}\right| .
$$

By virtue of the condition (2) and from results in (6), if $n \rightarrow \infty$, we can get $\left|\psi_{n}(t)-e^{i t}\right| \rightarrow 0$. The proof is complete.

Proof of Theorem 2.6. Evidently, the generating function of random variables $N_{n}$ is $g(t)=\frac{t\left(t^{n}-1\right)}{n(t-1)}$ and the characteristic function of random variables $X_{k}^{2}, k=$ $1,2, \ldots, n$ is $\varphi(t)=\frac{1}{\sqrt{1-2 i t}}$. Then, the characteristic function of random variable $\chi_{N_{n}}^{2} / n$ is given by

$$
\begin{aligned}
\varphi_{\frac{\chi_{N_{n}}^{2}}{n}}(t) & =g(\varphi(t / n))=\frac{\varphi(t / n)\left[\varphi^{n}(t / n)-1\right]}{n[\varphi(t / n)-1]} \\
& =\frac{\frac{1}{\sqrt{1-2 i t / n}}\left[\frac{1}{(1-2 i t / n)^{\frac{n}{2}}}-1\right]}{n\left[\frac{\sqrt{n}-\sqrt{n-2 i t}}{\sqrt{n-2 i t}}\right]}=\frac{(1+\sqrt{1-2 i t / n})\left[\frac{1}{(1-2 i t / n)^{\frac{n}{2}}}-1\right]}{2 i t} .
\end{aligned}
$$

In the proof of Theorem 2.1, we have

$$
\frac{1}{(1-2 i t / n)^{\frac{n}{2}}} \rightarrow e^{i t} \quad \text { as } n \rightarrow \infty \text {. }
$$

By letting $n \rightarrow \infty$, we can conclude that

$$
\varphi_{\frac{x_{N_{n}}^{2}}{n}}(t) \rightarrow \frac{e^{i t}-1}{i t}=\frac{e^{i t 1}-e^{i t 0}}{(1-0) i t} .
$$

This finishes the proof.

Proof of Theorem 2.7. Obviously, the generating functions of random variables $N_{n}$ is $g(t)=\frac{p_{n} t}{1-\left(1-p_{n}\right) t}$ and the characteristic function of random variables $X_{k}^{2}, k=1,2, \ldots, n$ is $\varphi(t)=\frac{1}{\sqrt{1-2 i t}}$. Then, the characteristic function of $p_{n} \cdot \chi_{N_{n}}^{2}$ is defined by

$$
\begin{aligned}
\varphi_{p_{n} \cdot \chi_{N_{n}}^{2}}(t) & =g\left(\varphi\left(p_{n} t\right)\right)=\frac{p_{n} \varphi\left(p_{n} t\right)}{1-\left(1-p_{n}\right) \varphi\left(p_{n} t\right)}=\frac{p_{n}}{\sqrt{1-2 i p_{n} t}-\left(1-p_{n}\right)} \\
& =\frac{p_{n}\left[\sqrt{1-2 i p_{n} t}+\left(1-p_{n}\right)\right]}{1-2 i p_{n} t-\left(1-p_{n}\right)^{2}}=\frac{\sqrt{1-2 i p_{n} t}+1-p_{n}}{2-2 i t-p_{n}} .
\end{aligned}
$$

By letting $n \rightarrow \infty$, we can assert that

$$
\varphi_{p_{n} \cdot \chi_{N_{n}}^{2}}(t) \rightarrow \frac{1}{1-i t}
$$

The proof is straightforward.

Proof of Theorem 2.8. According to assumptions of theorem and from Theorem 2.1, we have

$$
N_{n} \stackrel{d}{\rightarrow} \infty \quad \text { and } \quad \frac{\chi_{n}^{2}}{n} \stackrel{d}{\rightarrow} Z
$$


as $n \rightarrow \infty$. It is easily seen that

$$
\frac{\chi_{N_{n}}^{2}}{N_{n}} \stackrel{d}{\rightarrow} Z
$$

Because of the random variable $Z$ is generated at point one, we conclude

$$
\frac{\chi_{N_{n}}^{2}}{N_{n}} \stackrel{P}{\rightarrow} 1, \quad \text { as } \quad n \rightarrow \infty \text {. }
$$

Then, we can see that

$$
\frac{\chi_{N_{n}}^{2}}{n}=\frac{\chi_{N_{n}}^{2}}{N_{n}} \cdot \frac{N_{n}}{n} \stackrel{P}{\rightarrow} 1, \quad \text { as } \quad n \rightarrow \infty .
$$

Thus, the proof is complete

$$
\frac{\chi_{N_{n}}^{2}}{n} \stackrel{d}{\rightarrow} Z, \quad \text { as } \quad n \rightarrow \infty
$$

\section{Two approaches to computation of distribution functions of chi-square type $\chi_{N}^{2}$ with random degrees of freedom $N$.}

Firstly, using results of Lebedev in [6], we can construct an algorithm to find the values of distribution functions of chi-square type with random degrees of freedom $\chi_{N}^{2}(x)$.

Theorem 4.1 (We recall from [6]). Denote by $\chi_{n}^{2}(x)$ a distribution function of chi-square random variable $\chi_{n}^{2}$ with $n$ degrees of freedom. Then, for every $n=1,2, \ldots$,

with $\chi_{1}^{2}(x)=2 \Phi(\sqrt{x})-1, \quad \chi_{2}^{2}(x)=1-e^{-x / 2}$,

$$
\chi_{n+2}^{2}(x)=\chi_{n}^{2}(x)-\delta_{n} \frac{x^{n / 2}}{n ! !} e^{-x / 2}
$$

where

and

$$
\delta_{n}= \begin{cases}1, & n=2 k \\ \sqrt{\frac{2}{\pi}}, & n=2 k+1\end{cases}
$$

$$
\Phi(x)=\frac{1}{\sqrt{2 \pi}} \int_{-\infty}^{x} x^{-t / 2} d t .
$$

Corollary 4.2. If $n=2 m, m=1,2, \ldots$, then

$$
\chi_{2 m}^{2}(x)=1-e^{-x / 2} \sum_{p=0}^{m-1} \frac{x / 2^{p}}{p !} .
$$

If $n=2 m+1, m=0,1,2, \ldots$, then

$$
\chi_{2 m+1}^{2}(x)=2 \Phi(\sqrt{x})-1-\sqrt{\frac{2}{\pi}} e^{-x / 2} \sum_{p=1}^{m} \frac{x^{p-1 / 2}}{(2 p-1) ! !} .
$$


Theorem 4.3. Let $N$ be a positive integer-valued random variable with probability distribution $P(N=k)=p_{k}$. Assume that $N$ is independent from all $X_{i} \sim \mathcal{N}(0,1), i=1,2, \ldots$. Put $\chi_{n}^{2}=\sum_{i=1}^{n} X_{i}{ }^{2}$ and $\chi_{N}^{2}=\sum_{i=1}^{N} X_{i}{ }^{2}$. Denote by $\chi_{n}^{2}(x)$ and $F_{N}(x)$ the distribution functions of random variables $\chi_{n}^{2}$ and $\chi_{N}^{2}$. Then

$$
F_{N}(x)=\sum_{k=1}^{\infty} \chi_{k}^{2}(x) p_{k} .
$$

Proof. By virtue of formula of total probability and independence of $N$ for all $X_{i}, i=1,2, \ldots$, we have

$$
\begin{aligned}
F_{N}(x) & =P\left(\chi_{N}^{2} \leq x\right)=\sum_{k=1}^{\infty} P\left(\chi_{N}^{2} \leq x \mid N=k\right) P(N=k) \\
& =\sum_{k=1}^{\infty} P\left(\chi_{k}^{2} \leq x\right) P(N=k)=\sum_{k=1}^{\infty} \chi_{n}^{2}(x) p_{k}
\end{aligned}
$$

The proof is straightforward.

An algorithm for computing of distribution function $\chi_{N}^{2}(x)$ with random degrees of freedom

(1) Construct distribution function $\chi_{n}^{2}(x):=P\left(\chi_{n}^{2}<x\right)$ (based on result from Corollary 4.2).

(2) Compute $P(N=k)=p_{k}, k=1,2, \ldots$ of discrete random variable $N$.

(3) Compute distribution function of random variable $\chi_{N}^{2}$ (based on results from Theorem 4.3).

According to above algorithm, by using Maple, we can get computations of distribution functions of random variable $\chi_{N}^{2}$, where $N$ is a positive integervalued random variable. The received results from algorithm will be given in Appendix.

From now we can show another way for approaching to distribution functions of $\chi_{N}^{2}$ by directly computing its density function. Here, we compute the density function of $\chi_{N}^{2}$, where $N$ be a random variable from negative binomial law, i.e.,

$$
P(N=k)=C_{k-1}^{r-1} p^{r} q^{k-r} \quad(p+q=1, k=r, r+1, \ldots) .
$$

Let us consider the series

$$
\varphi(x)=\sum_{k=r}^{\infty} C_{k-1}^{r-1} \frac{x^{n}}{\Gamma\left(\frac{k}{2}\right)}
$$

with convergent domain $\mathbb{R}$. Let $f_{k}(x)$ be a density function of $\chi_{k}^{2}$ with $k$ degrees of freedom (see (1)). Then, by virtue of (7), it is easily seen that the random 
sum $\chi_{N}^{2}$ will have density function $f(x), x \geq 0$, denoted by

$$
\begin{aligned}
f(x) & =\sum_{k=r}^{\infty} \frac{x^{\frac{k}{2}-1} e^{-\frac{x}{2}}}{2^{\frac{k}{2}} \Gamma\left(\frac{k}{2}\right)} C_{k-1}^{r-1} p^{r} q^{k-r}=x^{-1} e^{-\frac{x}{2}}(p / q)^{r} \sum_{k=r}^{\infty} C_{k-1}^{r-1} \frac{[q \sqrt{x / 2}]^{k}}{\Gamma\left(\frac{k}{2}\right)} \\
& =x^{-1} e^{-\frac{x}{2}}(p / q)^{r} \varphi(q \sqrt{x / 2}) .
\end{aligned}
$$

Thus, we firstly must compute the series $\varphi(x)$ in $(8)$. Let us consider the series

$$
\phi_{n}(x)=\sum_{k=1}^{\infty} \frac{k^{n} x^{k}}{\Gamma\left(\frac{k}{2}\right)} \quad n=0,1,2, \ldots
$$

with following properties:

i. $\phi_{0}(x)=\frac{x}{\sqrt{\pi}}+2 x^{2} e^{x^{2}} \Phi(x \sqrt{2})$, where $\Phi(x)$ is Laplace's function.

ii. $\phi_{n+1}(x)=x \frac{\partial}{\partial x} \phi_{n}(x)$.

By virtue of two above properties and by using Maple, we can get the series $\phi_{n}(x)$. For example

$$
\begin{aligned}
& \phi_{1}(x)=\frac{x\left(1+2 x^{2}\right)}{\sqrt{\pi}}+4 x^{2}\left(1+x^{2}\right) e^{x^{2}} \Phi(x \sqrt{2}) \\
& \phi_{2}(x)=\frac{x\left(1+10 x^{2}+4 x^{4}\right)}{\sqrt{\pi}}+8 x^{2}\left(1+3 x^{2}+x^{4}\right) e^{x^{2}} \Phi(x \sqrt{2})
\end{aligned}
$$

Thus, if $r=1$ then $\varphi(x)=\phi_{0}(x)$. And if $r \geq 2$, then

$$
\varphi(x)=\frac{1}{(r-1) !} \sum_{k=r}^{\infty}(k-r+1)(k-r+2) \cdots(k-1) \frac{x^{k}}{\Gamma\left(\frac{k}{2}\right)} .
$$

And we can demonstrate $\varphi(x)$ through the series $\phi_{n}(x)$. For example

$$
\begin{array}{ll}
r=2, & \varphi(x)=\phi_{1}(x)-\phi_{0}(x) \\
r=3, & \varphi(x)=\frac{1}{2 !}\left[\phi_{2}(x)-3 \phi_{1}(x)+2 \phi_{0}(x)\right] \\
r=4, & \varphi(x)=\frac{1}{3 !}\left[\phi_{3}(x)-6 \phi_{2}(x)+11 \phi_{1}(x)-6 \phi_{0}(x)\right]
\end{array}
$$

The values of distribution function $F(x)=\int_{0}^{x} f(t) d t$ in some cases will be illustrated in Appendix.

Acknowledgements. The authors would like to take this opportunity to thank The National Fundamental Research Program 2006-2008 (MOST, Vietnam), The Asia Research Center (VNU, Vietnam) Grant 2007-2008 and the Korea for Advanced Studies for financial supporting in research project. 


\section{Appendix}

In this section, three tables concerning the distribution functions of random variable $\chi_{N}^{2}$ with random degrees of freedom $N$ are established.

Table 1. Distribution function of chi square-geometry with parameter

$$
p=1 / 2 \text {. }
$$

\begin{tabular}{|c|c|c|c|c|c|c|c|c|c|c|}
\hline$x$ & 0.00 & 0.01 & 0.02 & 0.03 & 0.04 & 0.05 & 0.06 & 0.07 & 0.08 & 0.09 \\
\hline 0.0 & 0.00000 & 03999 & 05651 & 06925 & 08006 & 08959 & 09818 & 10608 & 11354 & 12049 \\
\hline 0.1 & 2716 & 13349 & 13952 & 14525 & 15089 & 15632 & 16157 & 16663 & 17157 & 17643 \\
\hline 0.2 & 09 & 572 & 19021 & 60 & 19890 & 317 & 33 & 40 & 1538 & 21937 \\
\hline 0.5 & & & 23081 & & 23827 & & & 97 & 25 & 590 \\
\hline 0.4 & 0.25930 & 26267 & 26596 & 26927 & 27251 & 27572 & 27895 & 28211 & 28520 & 28829 \\
\hline 0.5 & 0.29141 & 29441 & 29745 & 30041 & 30338 & 30635 & 30922 & 31213 & 31503 & 31786 \\
\hline 0.6 & 0.32066 & 32348 & 32625 & 32901 & 33178 & 33447 & 33716 & 33985 & 34253 & 34512 \\
\hline 0.7 & 0.34777 & 35039 & 35297 & 35552 & 35809 & 36060 & 36318 & 6569 & 36815 & 37059 \\
\hline 0.8 & 37309 & 37555 & 37797 & 38042 & 38283 & 38517 & 38753 & 38992 & 39232 & 39459 \\
\hline 0.9 & 0.39694 & 39922 & 40152 & 40378 & 40609 & 40840 & 41061 & 41287 & 41506 & 11730 \\
\hline 1.0 & 949 & 42167 & 42390 & 42600 & 42814 & 43032 & 43247 & 43461 & 43671 & 43882 \\
\hline 1.1 & 388 & 44295 & 44507 & 44715 & 44922 & 45127 & 45327 & 45528 & 45728 & 45935 \\
\hline 1.2 & 0.46127 & 46329 & 46526 & 46726 & 46925 & 47115 & 47312 & 47508 & 47705 & 47892 \\
\hline 1.3 & 0.48082 & 48278 & 48462 & 48648 & 48837 & 49027 & 49209 & 49400 & 49577 & 49768 \\
\hline 1.4 & 0.49948 & 50131 & 50312 & 50488 & 50673 & 50856 & 51031 & 51210 & 51391 & 51560 \\
\hline 1.5 & 51729 & 51910 & 52 & 52259 & 52432 & 52605 & 52775 & 47 & 3113 & 53288 \\
\hline 1.6 & 3451 & 53623 & 53788 & 3958 & 54119 & 54282 & 54450 & 54607 & 54778 & 54942 \\
\hline 1.7 & 0.55100 & 55267 & 55429 & 55582 & 55742 & 55901 & 56058 & 56217 & 56373 & 56538 \\
\hline 1.8 & 0.56685 & 56845 & 56995 & 57152 & 57308 & 57457 & 57611 & 57760 & 57910 & 58066 \\
\hline 1.9 & 8217 & 58357 & 58503 & 58657 & 58802 & 58952 & 59102 & 59242 & 59385 & 59537 \\
\hline 2.0 & 0.59683 & 59821 & 59967 & 60104 & 60253 & 60397 & 60533 & 60675 & 60817 & 9955 \\
\hline 2.1 & 0.61090 & 61232 & 61373 & 61511 & 61648 & 61779 & 61919 & 62047 & 62187 & 62322 \\
\hline 2.2 & 0.62454 & 62595 & 62721 & 62859 & 62990 & 63122 & 63243 & 63378 & 63513 & 63642 \\
\hline 2.3 & 0.63774 & 63900 & 64031 & 64158 & 64282 & 64413 & 64537 & 64660 & 64787 & 64916 \\
\hline 2.4 & 35 & 61 & 65287 & 5 & 65533 & 11 & 65 & 99 & 66017 & 66135 \\
\hline 2. & 55 & 75 & 66498 & 66617 & 66734 & $68 \quad-$ & 668 & 7088 & 02 & 7320 \\
\hline 2.6 & 0.67439 & 67554 & 67662 & 67787 & 67894 & 68007 & 68125 & 68238 & 68352 & 6846 \\
\hline 2.7 & 0.68579 & 68678 & 68792 & 68905 & 69019 & 69126 & 69239 & 69339 & 69453 & 69558 \\
\hline 2.8 & 0.69668 & 69782 & 69887 & 69992 & 70099 & 70206 & 70307 & 70415 & 70521 & 70623 \\
\hline 2. & 0.7 & 70834 & 70940 & 71040 & 71143 & 71247 & 71350 & 71451 & 71553 & 71652 \\
\hline 3.0 & & 71851 & 949 & 72052 & 51 & 72248 & 2 & 51 & 5547 & 2641 \\
\hline 3.1 & 0.72736 & 72833 & 72930 & 73027 & 73123 & 73216 & 73310 & 73409 & 73501 & 73602 \\
\hline 3.2 & 0.73686 & 73783 & 73869 & 73973 & 74067 & 74153 & 74243 & 74339 & 74428 & 74516 \\
\hline 3.3 & 0.74609 & 74699 & 74787 & 74873 & 74968 & 75057 & 75149 & 75233 & 75318 & 75411 \\
\hline 3.4 & 96 & 2 & 672 & 75757 & 75848 & 5926 & 012 & 76101 & 76187 & 76266 \\
\hline 3.5 & & & 520 & & 76684 & 7677 & & 76936 & 77015 & 7102 \\
\hline 3.6 & 0.77176 & 77257 & 77344 & 77426 & 77503 & 77584 & 77665 & 77747 & 77824 & 77898 \\
\hline 3.7 & 0.77978 & 78057 & 78134 & 78210 & 78286 & 78365 & 78142 & 78523 & 78595 & 78673 \\
\hline 3.8 & 0.78751 & 78834 & 78894 & 78974 & 79050 & 79129 & 79193 & 79272 & 79353 & 79417 \\
\hline 3.9 & 97 & 79573 & 39 & 79715 & 3784 & 63 & 79929 & 80002 & 80069 & 80137 \\
\hline 4. & 215 & 80284 & 80357 & 80423 & 80492 & 80570 & 80632 & 80698 & 7772 & 1 \\
\hline 4.1 & 0.80906 & 80978 & 81045 & 81110 & 81173 & 81244 & 81308 & 81384 & 81438 & 81508 \\
\hline
\end{tabular}




\begin{tabular}{|c|c|c|c|c|c|c|c|c|c|c|}
\hline 4.2 & 0.81579 & 81642 & 81704 & 81774 & 81838 & 81900 & 81961 & 82038 & 82100 & 82161 \\
\hline 4.3 & 0.82220 & 82287 & 82344 & 82417 & 82476 & 82540 & 82601 & 82663 & 82718 & 82785 \\
\hline 4.4 & 0.82851 & 82905 & 82968 & 83032 & 83090 & 83155 & 83213 & 83276 & 83331 & 83392 \\
\hline 4.5 & 0.83449 & 83515 & 83569 & 83629 & 83683 & 83750 & 83809 & 83856 & 83917 & 83978 \\
\hline 4.7 & 0.84030 & 84095 & 84148 & 84198 & 84260 & 84318 & 84376 & 84425 & 84490 & 84542 \\
\hline 4.8 & 0.84597 & 84647 & 84709 & 84762 & 84819 & 84867 & 84923 & 84971 & 85028 & 85082 \\
\hline 4.9 & 0.85134 & 85194 & 85249 & 85303 & 85353 & 85406 & 85454 & 85513 & 85561 & 85613 \\
\hline 5.0 & 0.85666 & 85719 & 85763 & 85811 & 85866 & 85917 & 85974 & 86020 & 86065 & 86116 \\
\hline 5.1 & 0.86164 & 86210 & 86262 & 86311 & 86369 & 86410 & 86458 & 86509 & 86561 & 86609 \\
\hline 5.2 & 0.86651 & 86700 & 86756 & 86798 & 86841 & 86892 & 86937 & 86987 & 87034 & 87074 \\
\hline 5.3 & 0.87118 & 87178 & 87212 & 87266 & 87307 & 87351 & 87398 & 87445 & 87493 & 87531 \\
\hline 5.4 & 0.87584 & 87623 & 87671 & 87707 & 87760 & 87800 & 87845 & 87890 & 87935 & 87971 \\
\hline 5.5 & 0.88016 & 88061 & 88106 & 88149 & 88185 & 88234 & 88273 & 88324 & 88361 & 88395 \\
\hline 5.6 & 0.88447 & 88482 & 88532 & 88567 & 88609 & 88650 & 88694 & 88728 & 88770 & 88811 \\
\hline 5.7 & 0.88850 & 88893 & 88935 & 88976 & 89013 & 89052 & 89092 & 89131 & 89166 & 89203 \\
\hline 5.8 & 0.89252 & 89292 & 89327 & 89363 & 89396 & 89435 & 89483 & 89513 & 89552 & 89590 \\
\hline 5.9 & 0.89620 & 89660 & 89706 & 89745 & 89777 & 89816 & 89851 & 89888 & 89922 & 89956 \\
\hline 6.0 & 0.89992 & 90030 & 90080 & 90112 & 90141 & 90178 & 90214 & 90248 & 90278 & 90314 \\
\hline 6.1 & 0.90347 & 90386 & 90421 & 90456 & 90494 & 90524 & 90555 & 90591 & 90631 & 90660 \\
\hline 6.2 & 0.90694 & 90729 & 90764 & 90798 & 90832 & 90858 & 90894 & 90927 & 90960 & 90992 \\
\hline 6.3 & 0.91020 & 91059 & 91086 & 91126 & 91155 & 91179 & 91210 & 91249 & 91283 & 91314 \\
\hline 6.4 & 0.91346 & 91378 & 91414 & 91434 & 91466 & 91499 & 91532 & 91562 & 91593 & 91616 \\
\hline 6.5 & 0.91651 & 91685 & 91710 & 91739 & 91772 & 91802 & 91832 & 91861 & 91886 & 91913 \\
\hline 6.6 & 0.91947 & 91981 & 92004 & 92033 & 92063 & 92095 & 92112 & 92150 & 92180 & 92209 \\
\hline 6.7 & 0.92230 & 92259 & 92288 & 92316 & 92347 & 92377 & 92402 & 92429 & 92459 & 92486 \\
\hline 6.8 & 0.92506 & 92541 & 92562 & 92589 & 92616 & 92644 & 92673 & 92699 & 92726 & 92752 \\
\hline 6.9 & 0.92774 & 92806 & 92826 & 92859 & 92880 & 92912 & 92932 & 92958 & 92984 & 93017 \\
\hline 7 & 0.93284 & 93525 & 93757 & 93982 & 94188 & 94405 & 94602 & 94791 & 94983 & 95158 \\
\hline 8 & 0.95340 & 95505 & 95662 & 95812 & 95965 & 96119 & 96247 & 96382 & 96510 & 96633 \\
\hline 9 & 0.96758 & 96875 & 96990 & 97091 & 97197 & 97305 & 97399 & 97492 & 97584 & 97664 \\
\hline 10 & 0.97766 & 97839 & 97920 & 97988 & 98057 & 98130 & 98199 & 98265 & 98323 & 98389 \\
\hline 11 & 0.98448 & 98496 & 98555 & 98605 & 98654 & 98707 & 98754 & 98796 & 98844 & 98883 \\
\hline 12 & 0.98932 & 98963 & 99001 & 99030 & 99074 & 99107 & 99132 & 99171 & 99200 & 99230 \\
\hline 13 & 0.99254 & 99279 & 99311 & 99336 & 99355 & 99380 & 99401 & 99419 & 99451 & 99467 \\
\hline 14 & 0.99488 & 99500 & 99520 & 99536 & 99553 & 99570 & 99586 & 99606 & 99616 & 99636 \\
\hline 15 & 0.99645 & 99655 & 99669 & 99678 & 99690 & 99704 & 99714 & 99722 & 99732 & 99743 \\
\hline 16 & 0.99756 & 99760 & 99768 & 99777 & 99785 & 99800 & 99807 & 99816 & 99818 & 99830 \\
\hline 17 & 0.99831 & 99840 & 99842 & 99845 & 99851 & 99860 & 99863 & 99867 & 99871 & 99875 \\
\hline 18 & 0.99881 & 99884 & 99888 & 99893 & 99896 & 99902 & 99903 & 99906 & 99912 & 99912 \\
\hline 19 & 0.99917 & 99918 & 99924 & 99924 & 99927 & 99932 & 99933 & 99934 & 99935 & 99937 \\
\hline 20 & 0.99948 & 99942 & 99944 & 99946 & 99957 & 99950 & 99951 & 99953 & 99955 & 99956 \\
\hline
\end{tabular}

Table 2. Distribution function of chi square-negative binomial with parameter $(r=1, p=1 / 3)$.

Density function is

$$
f(x)= \begin{cases}0 & \text { if } x \leq 0 \\ p q e^{-x\left(1-q^{2}\right) / 2} \Phi(q \sqrt{x})+\frac{p e^{-x / 2}}{\sqrt{2 \pi x}} & \text { if } x>0\end{cases}
$$




\begin{tabular}{|c|c|c|c|c|c|c|c|c|c|c|}
\hline$x$ & 0.0 & 0.1 & 0.2 & 0.3 & 0.4 & 0.5 & 0.6 & 0.7 & 0.8 & 0.9 \\
\hline$\overline{0}$ & 0 & 0.0949 & 0.1401 & 0.1768 & 0.2089 & 0.2379 & 0.2647 & 0.2897 & 0.3132 & 0.3355 \\
\hline 1 & 0.3566 & 0.3768 & 0.3961 & 0.4146 & 0.4324 & 0.4494 & 0.4659 & 0.4817 & 0.4970 & 0.5117 \\
\hline 2 & 0.5260 & 0.5397 & 0.5531 & 0.5660 & 0.5784 & 0.5905 & 0.6022 & 0.6135 & 0.6245 & 0.6352 \\
\hline 3 & 0.6455 & 0.6555 & 0.6652 & 0.6747 & 0.6838 & 0.6927 & 0.7013 & 0.7097 & 0.7178 & 0.7257 \\
\hline 4 & 0.7334 & 0.7408 & 0.7480 & 0.7550 & 0.7619 & 0.7685 & 0.7749 & 0.7812 & 0.7872 & 0.7931 \\
\hline 5 & 0.7 & 0.8045 & 0.8099 & 0.8151 & 0.8202 & 0.8252 & 0.8300 & .8347 & 0.8393 & 0.8437 \\
\hline 6 & 0.8481 & 0.8523 & 0.8563 & 0.8603 & 0.8641 & 0.8679 & 0.8715 & 0.8751 & 0.8785 & 0.8819 \\
\hline 7 & 0.8851 & 0.8883 & 0.8913 & 0.8943 & 0.8972 & 0.9001 & 0.9028 & 0.9055 & 0.9081 & 0.9106 \\
\hline 8 & 0.9131 & 0.9155 & 0.9178 & 0.9200 & 0.9222 & 0.9244 & 0.9265 & 0.9285 & 0.9304 & 0.9323 \\
\hline 9 & 0.9342 & 0.9360 & 0.9378 & 0.9395 & 0.9411 & 0.9428 & 0.9443 & 0.9459 & 0.9473 & 0.9488 \\
\hline 10 & 0.9502 & 0.9516 & 0.9529 & 0.9542 & 0.9554 & 0.9567 & 0.9578 & 0.9590 & 0.9601 & 0.9612 \\
\hline 11 & 0.9623 & 0.9633 & 0.9643 & 0.9653 & 0.9663 & 0.9672 & 0.9681 & 0.9690 & 0.9698 & 0.9706 \\
\hline 12 & 0.9714 & 0.9722 & 0.9730 & 0.9737 & 0.9744 & 0.9751 & 0.9758 & 0.9765 & 0.9771 & 0.9778 \\
\hline 13 & 0.9784 & 0.9790 & 0.9795 & 0.9801 & 0.9806 & 0.9812 & 0.9817 & 0.9822 & 0.9827 & 0.9832 \\
\hline 14 & 0.9836 & .9841 & 0.9845 & 0.9849 & 0.9853 & 0.9857 & 0.9861 & 0.9865 & 0.9869 & 0.9872 \\
\hline 15 & 0.9876 & 0.9879 & 0.9883 & 0.9886 & 0.9889 & 0.9892 & 0.9895 & 0.9898 & 0.9901 & 0.9903 \\
\hline 16 & 0.9906 & 0.9909 & 0.9911 & 0.9914 & 0.9916 & 0.9918 & 0.9920 & 0.9923 & 0.9925 & 0.9927 \\
\hline 17 & 0.9929 & 0.9931 & 0.9933 & 0.9935 & 0.9936 & 0.9938 & 0.9940 & 0.9941 & 0.9943 & 0.9945 \\
\hline 18 & 0.9946 & 0.9948 & 0.9949 & 0.9950 & 0.9952 & 0.9953 & 0.9954 & 0.9956 & 0.9957 & 0.9958 \\
\hline 19 & 0.9959 & 0.9960 & 0.9961 & 0.9962 & 0.9963 & 0.9964 & 0.9965 & 0.9966 & 0.9967 & 0.9968 \\
\hline 20 & $0.9 \overline{969}$ & 0.9970 & 0.9971 & 0.9972 & $0 . \overline{9972}$ & 0.9973 & 0.9974 & 0.9975 & 0.9975 & 0.9976 \\
\hline 21 & 0.9977 & 0.9977 & 0.9978 & 0.9978 & 0.9979 & 0.9980 & 0.9980 & 0.9981 & 0.9981 & 0.9982 \\
\hline 22 & 0.9982 & 0.9983 & 0.9983 & 0.9984 & 0.9984 & 0.9985 & 0.9985 & 0.9985 & 0.9986 & 0.9986 \\
\hline 23 & .9987 & .9987 & 0.9987 & 0.9988 & 0.9988 & 0.9988 & 0.9989 & 0.9989 & 0.9989 & 0.9990 \\
\hline 24 & .9990 & 0.9990 & 0.9990 & 0.9991 & 0.9991 & 0.9991 & 0.9991 & 0.9992 & 0.9992 & 0.9992 \\
\hline 25 & 0.9992 & 0.9992 & 0.9993 & 0.9993 & 0.9993 & 0.9993 & 0.9993 & 0.9994 & 0.9994 & 0.9994 \\
\hline 26 & 0.9994 & 0.9994 & 0.9994 & 0.9995 & 0.9995 & 0.9995 & 0.9995 & 0.9995 & 0.9995 & 0.9995 \\
\hline 27 & 0.9996 & 0.9996 & 0.9996 & 0.9996 & 0.9996 & 0.9996 & 0.9996 & 0.9996 & 0.9996 & 0.9997 \\
\hline 28 & 0.9997 & 0.9997 & 0.9997 & 0.9997 & 0.9997 & 0.9997 & 0.9997 & 0.9997 & 0.9997 & .9997 \\
\hline 29 & 1997 & 0.9998 & 0.9998 & 0.9998 & 0.9998 & 0.9998 & 0.9998 & 0.9998 & 0.9998 & 0.9998 \\
\hline
\end{tabular}

Table 3. Distribution function of chi square-negative binomial with parameter $(r=2, p=1 / 3)$.

Density function is

$$
f(x)= \begin{cases}0 & \text { if } x \leq 0 \\ p^{2} e^{-x\left(1-q^{2}\right) / 2} \Phi(q \sqrt{x})\left(1+q^{2} x\right)+\frac{p^{2} q \sqrt{x} e^{-x / 2}}{\sqrt{2 \pi}} & \text { if } x>0\end{cases}
$$

\begin{tabular}{|c|c|c|c|c|c|c|c|c|c|c|}
\hline$x$ & 0.0 & 0.1 & 0.2 & 0.3 & 0.4 & 0.5 & 0.6 & 0.7 & 0.8 & 0.9 \\
\hline 0 & 0 & 0.0068 & 0.0147 & 0.0233 & 0.0324 & 0.0419 & 0.0517 & 0.0618 & 0.0722 & 0.0829 \\
1 & 0.0937 & 0.1046 & 0.1157 & 0.1269 & 0.1382 & 0.1495 & 0.1609 & 0.1724 & 0.1839 & 0.1954 \\
2 & 0.2069 & 0.2184 & 0.2298 & 0.2413 & 0.2527 & 0.2641 & 0.2754 & 0.2866 & 0.2978 & 0.3090 \\
3 & 0.3200 & 0.3310 & 0.3419 & 0.3527 & 0.3634 & 0.3740 & 0.3845 & 0.3949 & 0.4052 & 0.4154 \\
4 & 0.4255 & 0.4355 & 0.4453 & 0.4551 & 0.4647 & 0.4742 & 0.4836 & 0.4929 & 0.5021 & 0.5111 \\
5 & 0.5200 & 0.5289 & 0.5375 & 0.5461 & 0.5545 & 0.5629 & 0.5710 & 0.5791 & 0.5871 & 0.5949 \\
6 & 0.6026 & 0.6103 & 0.6177 & 0.6251 & 0.6324 & 0.6395 & 0.6465 & 0.6534 & 0.6602 & 0.6669 \\
7 & 0.6735 & 0.6799 & 0.6863 & 0.6925 & 0.6987 & 0.7047 & 0.7106 & 0.7165 & 0.7222 & 0.7278 \\
\hline
\end{tabular}




\begin{tabular}{|c|c|c|c|c|c|c|c|c|c|c|}
\hline 8 & 0.7333 & 0.7388 & 0.7441 & 0.7493 & 0.7545 & 0.7595 & 0.7645 & 0.7693 & 0.7741 & 0.7788 \\
9 & 0.7834 & 0.7879 & 0.7923 & 0.7967 & 0.8009 & 0.8051 & 0.8092 & 0.8132 & 0.8172 & 0.8211 \\
10 & 0.8248 & 0.8286 & 0.8322 & 0.8358 & 0.8393 & 0.8427 & 0.8461 & 0.8494 & 0.8527 & 0.8558 \\
11 & 0.8589 & 0.8620 & 0.8650 & 0.8679 & 0.8708 & 0.8736 & 0.8763 & 0.8790 & 0.8817 & 0.8843 \\
12 & 0.8868 & 0.8893 & 0.8917 & 0.8941 & 0.8964 & 0.8987 & 0.9010 & 0.9032 & 0.9053 & 0.9074 \\
13 & 0.9095 & 0.9115 & 0.9134 & 0.9154 & 0.9173 & 0.9191 & 0.9209 & 0.9227 & 0.9244 & 0.9261 \\
14 & 0.9278 & 0.9294 & 0.9310 & 0.9325 & 0.9341 & 0.9356 & 0.9370 & 0.9384 & 0.9398 & 0.9412 \\
15 & 0.9425 & 0.9438 & 0.9451 & 0.9464 & 0.9476 & 0.9488 & 0.9500 & 0.9511 & 0.9522 & 0.9533 \\
16 & 0.9544 & 0.9554 & 0.9565 & 0.9575 & 0.9584 & 0.9594 & 0.9603 & 0.9612 & 0.9621 & 0.9630 \\
17 & 0.9639 & 0.9647 & 0.9655 & 0.9663 & 0.9671 & 0.9679 & 0.9686 & 0.9693 & 0.9701 & 0.9708 \\
18 & 0.9714 & 0.9721 & 0.9727 & 0.9734 & 0.9740 & 0.9746 & 0.9752 & 0.9758 & 0.9764 & 0.9769 \\
19 & 0.9775 & 0.9780 & 0.9785 & 0.9790 & 0.9795 & 0.9800 & 0.9805 & 0.9809 & 0.9814 & 0.9818 \\
\hline 20 & 0.9822 & 0.9827 & 0.9831 & 0.9835 & 0.9839 & 0.9842 & 0.9846 & 0.9850 & 0.9853 & 0.9857 \\
21 & 0.9860 & 0.9864 & 0.9867 & 0.9870 & 0.9873 & 0.9876 & 0.9879 & 0.9882 & 0.9885 & 0.9887 \\
22 & 0.9890 & 0.9893 & 0.9895 & 0.9898 & 0.9900 & 0.9903 & 0.9905 & 0.9907 & 0.9910 & 0.9912 \\
23 & 0.9914 & 0.9916 & 0.9918 & 0.9920 & 0.9922 & 0.9924 & 0.9926 & 0.9927 & 0.9929 & 0.9931 \\
24 & 0.9932 & 0.9934 & 0.9936 & 0.9937 & 0.9939 & 0.9940 & 0.9942 & 0.9943 & 0.9944 & 0.9946 \\
25 & 0.9947 & 0.9948 & 0.9950 & 0.9951 & 0.9952 & 0.9953 & 0.9954 & 0.9955 & 0.9957 & 0.9958 \\
26 & 0.9959 & 0.9960 & 0.9961 & 0.9962 & 0.9963 & 0.9963 & 0.9964 & 0.9965 & 0.9966 & 0.9967 \\
27 & 0.9968 & 0.9968 & 0.9969 & 0.9970 & 0.9971 & 0.9971 & 0.9972 & 0.9973 & 0.9974 & 0.9974 \\
28 & 0.9975 & 0.9975 & 0.9976 & 0.9977 & 0.9977 & 0.9978 & 0.9978 & 0.9979 & 0.9979 & 0.9980 \\
29 & 0.9980 & 0.9981 & 0.9981 & 0.9982 & 0.9982 & 0.9983 & 0.9983 & 0.9983 & 0.9984 & 0.9984 \\
\hline
\end{tabular}

\section{References}

[1] W. Feller, An Introduction to Probability Theory and Its Applications, vol II, 2nd edition, John Wiley and Sons, New York, 1971.

[2] N. L. Johnson and S. Kotz, Continuous Univariate Distribution-2, A Wiley-interscience publication, 1970.

[3] R. V. Hogg, J. W. McKean, and A. T. Craig, Introduction to Mathematical Statistics, Sixth Edition, International Edition, 2005.

[4] L. Hung Tran and T. T Tran, Some results on random sum of independent random variables, Submitted to Statistics and Probability Letters, 2007.

[5] L. H. Tran, T. T. Tran, and Q. V. Bui, On distribution function of chi-square with random degrees of freedom, (in vietnamese), to appear in Vietnam Journal of Applied Mathematics, 2007.

[6] E. A. Lebedev, On new properties distributions of mathematical statistics, Probability Theory, Random Processes, Mathematical Statistics and Applications, Proceedings of the Intertional Conference in honor of 70 years Jubilee of Professor, Doctor of Physical and Mathematical Sciences Gennady Medvedev, Minsk February 21-25, 2005, pp. 154160 .

[7] $\mathrm{H}$. Robbins, The asymptotic distribution of the sum of a random number of random variables, Bull. Amer. Math. Soc. 54 (1948), 1151-1161.

[8] Y. B. Shvetsov, Random sum estimators and their efficiency, Technical Report, Department of Mathematical Sciences, Montana State University, 2004, pp.1-20. 
TRAN LOC HunG

Department of Mathematics

HUE UNIVERSITY

VIETNAM

E-mail address: tlhungun@gmail.com

Tran THIEN THanh

Department of Mathematics

HUE UNIVERSITY

VIETNAM

BUI QUANG Vu

DEPARTMENT OF MATHEMATICS

HUE UNIVERSITY

VIETNAM 